\title{
Supra-Pubic Single Incision Cholecystectomy
}

\author{
Monika E. Hagen • Oliver J. Wagner • \\ Kari Thompson • Garth Jacobsen • Adam Spivack • \\ Brian Wong • Mark Talamini • Santiago Horgan
}

Received: 8 June 2009 / Accepted: 26 October 2009 / Published online: 12 November 2009

(C) 2009 The Author(s). This article is published with open access at Springerlink.com

\begin{abstract}
Introduction Surgery is moving towards less invasive and cosmetically superior approaches such as single incision laparoscopy (SIL). While trans-umbilical SIL is gaining popularity, incisions may lead to post-operative deformations of the umbilicus and the possibility of an increased rate of incisional hernias. Access within the pubic hairline allows preservation of the umbilicus and results in a scar which is concealed within the pubic hair.

Methods Supra-pubic single incision cholecystectomy was performed in a 30-year-old patient with symptomatic gallstones. A $2.5-\mathrm{cm}$ transverse incision was placed within the pubic hairline and a subcutaneous tunnel was formed. Three 5-mm ports were introduced into the tunnel and perforated the anterior rectus sheath superior to the skin incision. The surgical procedure was then undertaken with conventional laparoscopic instrumentation. The adjacent 5-mm incisions were merged for gallbladder removal. The entry site was closed under direct vision.

Results The above procedure was technically feasible and without complication. Operative time was $45 \mathrm{~min}$, and the patient was discharged $5 \mathrm{~h}$ post-operatively.

Conclusions Supra-pubic single incision laparoscopic cholecystectomy may offer a more cosmetically appealing result than standard umbilical access. The operation can be performed by surgeons skilled in single incision techniques with good result.

Keywords Single access surgery ·

Minimally invasive surgery $\cdot$ Laparoscopy $\cdot$ NOTES .

Single incision laparoscopic surgery

\section{Introduction}

Laparoscopic cholecystectomy is currently the gold standard for the treatment of symptomatic gallstones. ${ }^{1,2}$ Besides

Electronic supplementary material The online version of this article (doi:10.1007/s11605-009-1079-0) contains supplementary material, which is available to authorized users.

M. E. Hagen $(\triangle) \cdot$ O. J. Wagner $\cdot$ K. Thompson $\cdot$ G. Jacobsen •

A. Spivack $\cdot$ B. Wong $\cdot$ M. Talamini $\cdot$ S. Horgan

Department of Surgery, Center for the Future of Surgery,

University of California,

200 West Arbor Drive,

San Diego, CA 92108, USA

e-mail: MonikaHagen@aol.com

the numerous clinical advantages of laparoscopy over an open approach, recent surveys have demonstrated that patients clearly favor cosmetically superior approaches to the abdominal cavity. ${ }^{3}$ Less invasive and cosmetically superior approaches such as single incision laparoscopy (SIL) and natural orifice translumenal endoscopic surgery (NOTES) have been gaining popularity. While pure NOTES will result in cosmetically ideal outcomes, this method is still under development and is currently only performed at specialized centers. ${ }^{4,5}$ While we at UCSD have been actively involved in leading the surgical community towards the safe and proficient development of natural orifice techniques, we realize that there is a need to investigate all approaches which may improve the outcomes of surgical patients. Within the past year, single incision laparoscopic surgery has been described for a great variety of procedures with rapidly growing numbers. ${ }^{6-12}$ Single incision laparoscopic surgery is performed with slightly modified but still conventional rigid laparoscopic instruments, and results in less scaring when compared to
\end{abstract}


conventional laparoscopy. Still, single incision laparoscopic surgery requires an abdominal wall incision, usually in the umbilical area, and can lead to all the common problems of conventional laparoscopy particularly pain and hernia formation. ${ }^{1}$ Because of the increased size of the umbilical incision during single incision laparoscopy, the possibility of a growing rate of incisional hernias may be anticipated. Additionally, placing a $15 \mathrm{~mm}$ or larger incision in the umbilical area might lead to an umbilical deformation depending on technique, original shape, size, and other factors. Finally, many publications in the plastic surgery literature indicate the importance of the integrity of the umbilicus for the overall physical appearance. ${ }^{13-15}$ Suprapubic single incision surgery is a novel method for minimal invasive access procedures that preserves the native umbilicus, results in a more discrete scar, and has the potential to reduce the incidence of postoperative hernias.

\section{Material and Methods}

Extensive experience with single incision laparoscopic surgery prior to the first human case was gathered in the porcine model and human cadavers using different methods for access (trocars, open approach, and rigid and flexible instrumentation). The initial patient was listed under the institute's Institutional Review Board protocol for single incision laparoscopy. Possible advantages and complications of this new investigational method, and the possibility of conversion to conventional (laparoscopic or open) surgery were discussed with the patient and appropriate informed consent was given.

The patient was placed in reversed Trendelenburgposition and the bladder catheterized. A $2.5-\mathrm{cm}$ transverse incision was placed within the pubic hairline medially. A subcutaneous tunnel was formed in an open fashion in a cephalad direction of about $5 \mathrm{~cm}$ in length and the anterior rectus sheath visualized. Next a 5-mm optiview optical bladeless trocar (Endopath Xcel, Ethicon Endo-Surgery Inc., Cincinnati, OH, USA) with an introduced utilizing a 0-degree, 5-mm camera was guided through the subcutaneous tunnel followed by a stepwise passage through the abdominal wall under constant direct visualization. Pneumoperitoneum was then obtained and trocar position was verified. Next, two additional 5-mm low profile trocars (Ethicon Endo-Surgery Inc., Cincinnati, OH, USA), were inserted in a similar fashion, one on each side of the first trocar (Fig. 1). A conventional 5-mm rigid laparoscopic grasper was inserted through the left low profile trocar to retract the gallbladder at the fundus. Dissection of Calot's triangle was achieved with a harmonic scalpel (Ethicon Endo-Surgery Inc., Cincinnati, OH, USA) introduced through the right-sided trocar. Flexible laparoscopic instru-

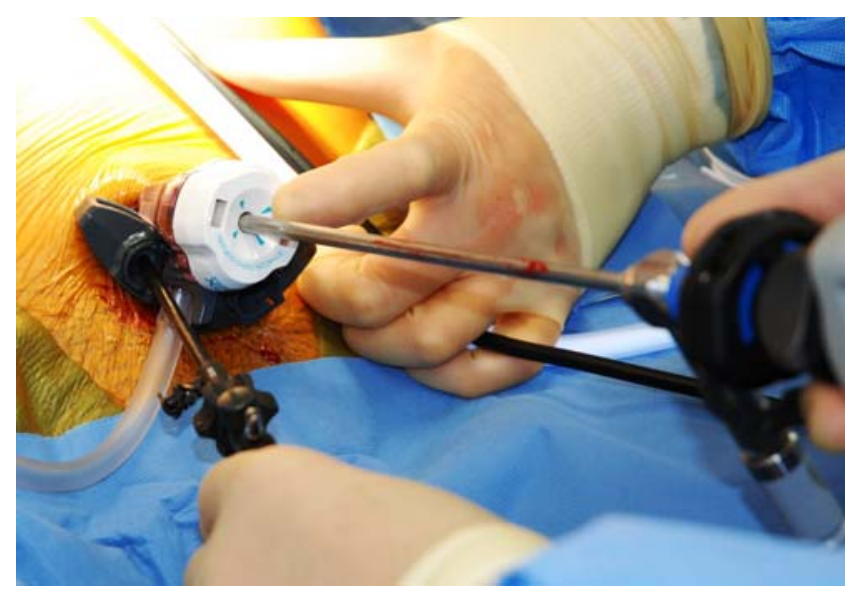

Figure 1 Setup of trocars.

ments were used for dissection when necessary (Real Hand, Novare Systems Inc., Cupertino, CA, USA). After obtaining a clear critical view, the cystic duct was secured using two distal Hem-o-lock clips (Weck Closure Systems, Research Triangle Park, NC, USA) and one proximal titanium clip (Ethicon Endo-Surgery Inc., Cincinnati, OH, USA). The cystic duct was divided with laparoscopic scissors. Then, the gallbladder was dissected free of the liver bed using alternating retraction from the left-sided grasper and the harmonic scalpel. An Endo-loop was then introduced through the right trocar and placed around the infundibulum of the gallbladder. All trocars were removed and the three adjacent trocar incisions were joined to facilitate removal of the gallbladder. The abdominal wall and the subcutaneous tunnel were closed in layers under direct visualization with interrupted 2-0 Vicryl sutures. The skin incision was closed in a subcuticular fashion (Fig. 2).

Surgical technique: Please see corresponding video.

\section{Results}

The patient was a 30-year-old female $(\mathrm{BMI}=24)$ with symptomatic uncomplicated gallstone disease and no previous abdominal surgery.

The above described procedure was feasible using conventional rigid and articulating laparoscopic instruments. Time to create the tunnel and achieve abdominal access was approximately $4 \mathrm{~min}$.

Gallbladder retraction was sufficient, and could provide adequate exposure by alternating the grasping site between the infundibulum and fundus of the gallbladder as needed. Visualization was as good as conventional laparoscopic view, and all critical structures were visualized without difficulty. Operative time was $45 \mathrm{~min}$. No complications occurred during the operation, and the immediate postop- 


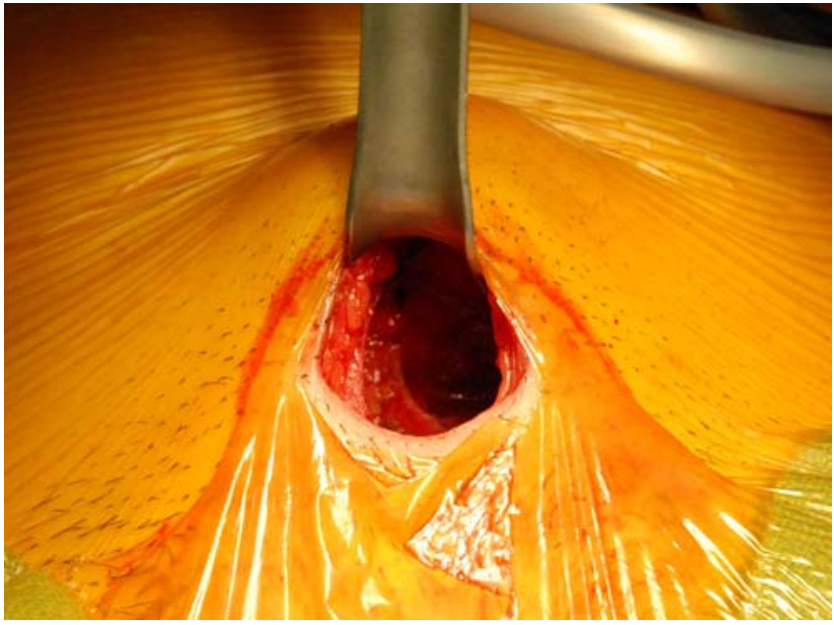

Figure 2 Subcutaneous tunnel before closure.

erative course was uncomplicated. The patient was discharged $5 \mathrm{~h}$ after the procedure. Evaluation 3 weeks after the operation showed an uncomplicated course and a clean scar within the re-growing pubic hair (Fig. 3).

\section{Discussion}

We describe a new surgical technique for single incision laparoscopic surgery applied in a patient. Supra-pubic single incision cholecystectomy was feasible in this case using a combination of conventional rigid and articulated laparoscopic instruments. This new method delivers a linear scar that can be hidden within the supra-pubic hairline and might therefore appear cosmetically superior when compared to scars after conventional laparoscopy and single incision transumbilical laparoscopy. In addition, the rate of incisional hernias for port sites which avoid the midline is significantly smaller when compared with those placed in the midline. ${ }^{16}$ After skin incision access through the abdominal wall can be chosen independent of the location of the skin incision, by creating a subcutaneous tunnel. Therefore, perforation through the rectal muscle instead of the linea alba can be performed easily in order to potentially avoid incisional hernias.

A limitation of this technique seems to lie in the entrapment of trocars in the subcutaneous tunnel and the entry into the abdominal cavity. Parallelism of trocars and limited range of motion of trocars lead to a loss of triangulation, a reduced field of surgery and intermittent conflict of instruments and camera on the outside. However, the use of articulated instruments was helpful to overcome some of these limitations by creating additional degrees of freedom. Still, the access to the abdominal cavity seems to represent the most critical challenge during this new method. Inserting a trocar through a preformed tunnel to enter the abdominal cavity in an angle smaller than $90^{\circ}$ is more difficult than entering in the usual vertical fashion. Incidental injuries might occur easier despite the visual control obtained by the camera inside the port. Additionally, while using optical trocars for laparoscopic access has been reported in great numbers in the upper abdomen ${ }^{17-19}$, only few reports can be found about their use in the lower abdomen and safety in that anatomical region might be reduced. ${ }^{19}$ Despite this uncertainty, a Foley catheter should be positioned during any supra-pubic access case to minimize the risks of any inadvertent bladder injuries.

New ports with extendable tips might offer solutions for this access. Also, flexible endoscopes might facilitate the passage through the subcutaneous tunnel and could be applied especially for longer and/or curved tunnels or be used in combination with laparoscopic instruments. In that sense, multiple tunnels can be placed to enter the abdominal cavity through a single skin incision. Different types of instruments could potentially be inserted to cover various aspects of abdominal surgery such as retraction, vision, dissection, and assistance. In that sense, this initial case only represents the feasible variation of a new surgical method and further research will be conducted to advance this technique.

\section{Conclusions}

Supra-pubic single incision laparoscopic cholecystectomy appeared feasible in our initial case. This new technique potentially offers less visible scaring (concealable in pubic hair) while maintaining an access through a single incision at a stable part the abdominal wall in order to reduce the risks for incisional hernias.

Whether this approach turns out to be superior to conventional laparoscopy, single incision laparoscopy and

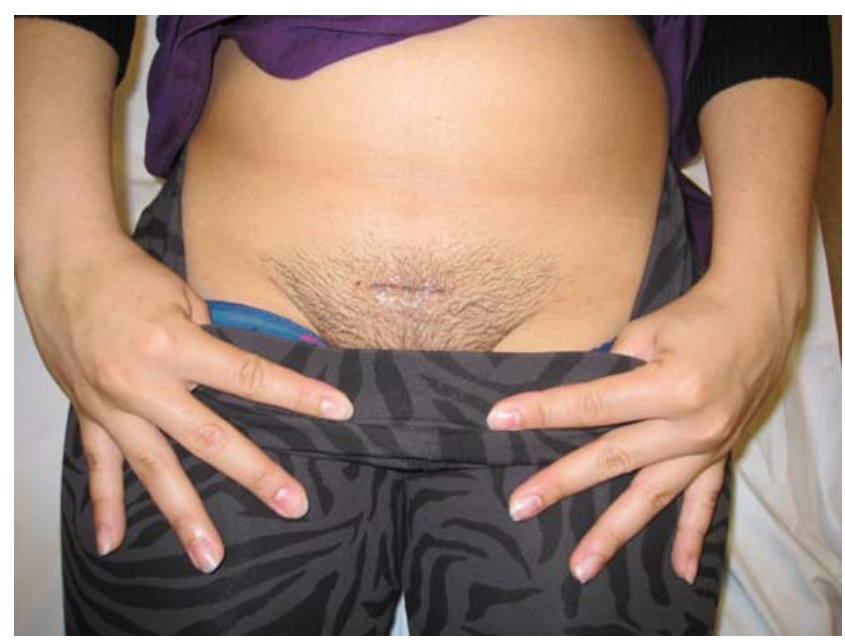

Figure 3 Scar 3 weeks postoperatively. 
NOTES regarding clinical parameters remains subject to more substantial research.

Open Access This article is distributed under the terms of the Creative Commons Attribution Noncommercial License which permits any noncommercial use, distribution, and reproduction in any medium, provided the original author(s) and source are credited.

\section{References}

1. Purkayastha S, Tilney HS, Georgiou P, Athanasiou T, Tekkis PP, Darzi AW. Laparoscopic cholecystectomy versus mini-laparotomy cholecystectomy: a meta-analysis of randomised control trials. Surg Endosc 2007;21:1294-1300.

2. Keus F, Werner JE, Gooszen HG, Oostvogel HJ, van Laarhoven CJ. Randomized clinical trial of small-incision and laparoscopic cholecystectomy in patients with symptomatic cholecystolithiasis: primary and clinical outcomes. Arch Surg 2008;143:371-377.

3. Hagen ME, Wagner OJ, Christen D, Morel P. Cosmetic issues of abdominal surgery: results of an enquiry into possible grounds for a natural orifice transluminal endoscopic surgery (NOTES) approach. Endoscopy 2008;40:581-583.

4. Swain P. A justification for NOTES-natural orifice translumenal endosurgery. Gastrointest Endosc 2007;65:514-516.

5. Giday SA, Magno P, Kalloo AN. NOTES: the future. Gastrointest Endosc Clin N Am 2008;18:387-395.

6. Gettman MT, Box G, Averch T et al. Consensus statement on natural orifice transluminal endoscopic surgery and single-incision laparoscopic surgery: heralding a new era in urology. Eur Urol 2008;53:1117-1120.

7. Raman JD, Cadeddu JA, Rao P, Rane A. Single-incision laparoscopic surgery: initial urological experience and comparison with natural-orifice transluminal endoscopic surgery. BJU Int 2008;101:1493-1496.
8. Jackson CC, Chwals WJ, Frim DM. A single-incision laparoscopic technique for retrieval and replacement of disconnected ventriculoperitoneal shunt tubing found in the peritoneum. Pediatr Neurosurg 2002;36:175-177.

9. Chang YT, Wang JY, Lee JY, Chiou CS, Hsieh JS. One-trocar laparoscopic transperitoneal closure of inguinal hernia in children. World J Surg 2008;32:2459-2463.

10. Ghezzi F, Cromi A, Fasola M, Bolis P. One-trocar salpingectomy for the treatment of tubal pregnancy: a 'marionette-like' technique. BJOG 2005;112:1417-1419.

11. Ghezzi F, Cromi A, Fasola M, Bolis P. One-trocar salpingectomy for the treatment of tubal pregnancy: a 'marionette-like' technique. BJOG 2005;112:1417-1419.

12. Lima M, Tursini S, Ruggeri G, Gargano T, Libri M, Domini M. One trocar assisted pyeloplasty (OTAP): initial experience and codification of a technique. Pediatr Med Chir 2007;29:108-111.

13. Barbosa MV, Nahas FX, Ferreira LM. A practical dressing to the umbilical stalk. J Plast Reconstr Aesthet Surg 2008;61:851852.

14. Dini GM, Ferreira LM. A simple technique to correct umbilicus vertical malposition. Plast Reconstr Surg 2007;119:1973-1974.

15. Jardini Barbosa MV, Nahas FX, Sabia Neto MA, Ferreira LM. Strategies in umbilical reconstruction. J Plast Reconstr Aesthet Surg 2008;62:e147-e150.

16. Montz FJ, Holschneider $\mathrm{CH}$, Munro MG. Incisional hernia following laparoscopy: a survey of the American Association of Gynecologic Laparoscopists. Obstet Gynecol 1994;84:881-884.

17. Rabl C, Palazzo F, Aoki H, Campos GM. Initial laparoscopic access using an optical trocar without pneumoperitoneum is safe and effective in the morbidly obese. Surg Innov 2008;15:126-131.

18. Berch BR, Torquati A, Lutfi RE, Richards WO. Experience with the optical access trocar for safe and rapid entry in the performance of laparoscopic gastric bypass. Surg Endosc 2006;20:1238-1241.

19. String A, Berber E, Foroutani A, Macho JR, Pearl JM, Siperstein AE. Use of the optical access trocar for safe and rapid entry in various laparoscopic procedures. Surg Endosc 2001;15:570-573. 